11

\title{
Особенности нагрева волноводов при передаче сверхвысокочастотных сигналов большой мощности
}

\author{
() И.В. Кудрявцев, О.Б. Гоцелюк, Е.С. Новиков, В.Г. Дёмин \\ Сибирский фредеральный университет, \\ 660041 Красноярск, Россия \\ e-mail: ikudryavcev@sfu-kras.ru
}

(Поступило в Редакцию 29 декабря 2015 г. В окончательной редакции 16 июня 2016 г.)

Исследовано явление нагрева волноводов при передаче СВЧ сигналов. Для оценки тепловыделения разработаны математические модели и получены дифференциальные уравнения теплового баланса, учитывающие различные условия эксплуатации волноводов. Полученные результаты свидетельствуют о необходимости дальнейшего исследования влияния тепловыделения в волноводах на их прочностные и функциональноэксплуатационные параметры.

DOI: 10.21883/JTF.2017.01.44024.1724

\section{Введение}

Волноводы являются частью антенно-фидерной системы (АФС) и служат для передачи электромагнитных сигналов сверхвысокой частоты (СВЧ) между антеннами и приемо-передающими блоками в различной технике, например в космических аппаратах. В процессе эксплуатации волноводы подвергаются различным воздействиям в виде деформационных и силовых статических и динамических нагрузок, а также циклическим температурным воздействиям от солнечного излучения и передачи высокочастотных сигналов, которые оказывают влияние на функционально-эксплуатационные параметры АФС в целом [1].

Для обеспечения требуемой прочности и жесткости волноводов, разработаны соответствующие методики их расчета на деформационные и силовые внешние воздействия $[2,3]$. Однако все возрастающие требования к улучшению функционально-эксплуатационных параметров всех систем космических аппаратов, в частности к антенно-фидерным системам, приводит к необходимости повышения мощности передаваемых сигналов по волноводам при одновременном увеличении их срока активного существования до 15 и более лет. Это требует уточнения существующих методов расчета волновода для учета влияния тех внешних факторов, которые ранее считались второстепенными.

Например, наземные испытания АФС, проведенные в АО „Информационные спутниковые системы им. М.Ф. Решетнева“, показали, что при передаче высокочастотных сигналов повышенной мощностью, $10 \mathrm{~kW}$ и более, происходит нагрев волноводов до $40-60^{\circ} \mathrm{C}$. Поскольку в течение эксплуатации КА на орбите передача сигналов высокой мощности происходит регулярно, данный нагрев будет представлять собой дополнительное термоциклирование, которое необходимо учитывать в составе периодического нагрева от солнечного излучения.

Количество циклов нагрева от передачи СВЧ-сигналов значительно превышает число циклов термического на- гружения в результате действия солнечного излучения и в сумме они могут превысить базовое число циклов, соответствующих пределу выносливости материала конструкции волновода, что делает актуальной задачу расчета и на усталостную прочность.

Различие в механических (модуль упругости, коэффициент Пуассона и др.) и тепловых (КТР, теплоемкость и др.) свойствах материала конструкции волновода (алюминий, медь, сплавы) и нанесенного на его внутренние стенки токопроводящего покрытия (золото, серебро, медь и др.) приводит к появлению в местах их контакта касательных напряжений, которые будут изменяться во времени по периодическому закону в соответствии с периодичностью нагрева при передаче сигналов.

Также в результате термоциклирования может произойти недопустимое ухудшение свойств материала стенок волновода с образованием микротрещин, что, в сочетании с периодическим воздействием касательных напряжений может привести к отслаиванию слоя токопроводящего материала и даже нарушению герметичности его конструкции [4].

Ситуация осложняется тем, что возможности для рассеивания избыточного тепла в условиях открытого космоса весьма ограничены, особенно при негерметичном исполнении космического аппарата, когда все его элементы и системы подвергаются воздействию солнечного излучения, а конвективный обмен практически отсутствует. Все это затрудняет эффективный отвод тепла и требует выполнения расчетов с учетом всех факторов, включая и нагрев при передаче сигналов по волноводам.

\section{1. Используемые ранее подходы к моделированию}

Проведенный аналитический обзор существующей литературы [5-9] по данной проблеме показал, что авторы рассмотренных источников отмечают только лишь очень малые потери передаваемых сигналов в волноводах по 
сравнению с другими типами направляющих систем и как следствие ими делается вывод об отсутствии необходимости учета выделения тепла.

Считаем, что такая ситуация является допустимой только для СВЧ сигналов небольшой мощности, однако при передаче сигналов большой мощности этим нагревом пренебрегать нельзя по вышеуказанным причинам. В связи с этим необходима доработка методов прочностного расчета тонкостенной конструкции волноводов, изложенных в [1,2,3], на предмет учета периодического нагрева, образующегося при передаче сигналов повышенной мощности.

\section{1. Потери мощности сигнала}

Анализ литературных источников [5-9] показал, что в процессе распространения электромагнитной волны по волноводу происходит затухание передаваемого сигнала вследствие рассеивания части его энергии по тонкостенной конструкции. Причин затухания сигнала несколько:

1) потери в электропроводящем слое материала на внутренних стенках волновода,

2) потери в диэлектрике во внутренней полости волновода,

3) потери на излучение из-за неплотного соединения отдельных элементов участка волноводнораспределительной системы и др.

В большинстве встречающихся на практике случаев преобладающими являются потери в тонком электропроводящем слое на внутренних стенках волновода $[5,6]$. В теории волноводов [7,8] принимается идеальная электрическая проводимость его материала, при которой электрические силовые линии будут строго перпендикулярны к стенкам конструкции волновода.

В реальных материалах вследствие наличия у них электрического сопротивления вектор напряженности электрического поля будет наклонен в сторону распространения сигнала, и у этого поля появится тангенциальная составляющая. Отклонение электрических силовых линий приводит к изменению и магнитного поля - у внутренних стенок волновода появится нормальная составляющая вектора напряженности магнитного поля, которая в случае идеального

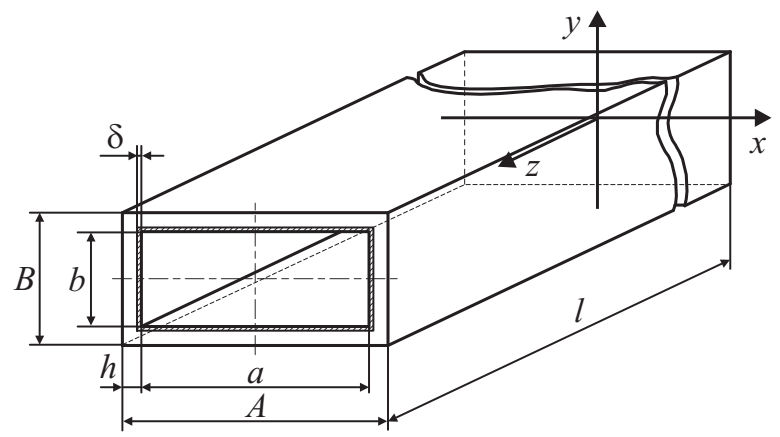

Рис. 1. Прямой элемент волновода. материала волновода равнялась нулю. Вследствие такого изменения структуры электромагнитного поля вектор Пойтинга будет иметь компоненту, направленную перпендикулярно к стенкам волновода, и наведенные токи будут проходить не только по их поверхностям, а проникать на некоторую глубину $\delta$, называемую скинслоем, и за счет наличия у материала электрического сопротивления преобразовываться в джоулево тепло.

В результате этого все составляющие электромагнитного поля вдоль осевой линии волновода (ось $z$ на рис. 1) будут уменьшаться по экспоненциальному закону [9], согласно которому уменьшение потока энергии вдоль волновода можно охарактеризовать изменением мощности $\Delta P$ передаваемого по нему сигнала:

$$
\Delta P=P_{\text {in }}-P_{\text {out }}=P_{\text {in }}-P_{\text {in }} e^{-2 \alpha l}=P_{i}\left(1-e^{-2 \alpha l}\right)[\mathrm{W}],
$$

где $P_{\text {in }}-$ мощность на входе волновода, $\mathrm{W} ; P_{\text {out }}-$ мощность на выходе волновода, $\mathrm{W} ; \alpha-$ коэффициент потерь, $\mathrm{dB} / \mathrm{m} ; l$ - длина волновода, $\mathrm{m}$.

Согласно выражению (1), потери мощности сигнала $\Delta P$ в волноводе можно охарактеризовать с помощью коэффициента потерь (затухания) $\alpha$, которым и принято оценивать степень рассеяния электромагнитной энергии при распространении ее вдоль волновода с учетом всех возможных причин этого явления.

\section{2. Коэффициент потерь}

Значение коэффициента потерь $\alpha$ можно определить опытным путем (эмпирически) либо с помощью теоретических зависимостей [5-9], которые учитывают условия распространения сигнала: тип и размеры волновода, свойства материала его стенок и скин-слоя, наличие/отсутствие диэлектрика, характеристики передаваемой по нему электромагнитной волны и др.

Например, в наиболее распространенном случае передачи по волноводу прямоугольного поперечного сечения магнитной волны типа $H_{10}$ теоретическое значение коэффициента потерь $\alpha$, согласно [5], можно определить по следующей зависимости:

$$
\alpha=10^{-3} \sqrt{0.1 \pi f \mu_{\alpha} \rho} \frac{1+\frac{2 b}{a}\left(\frac{\lambda}{2 a}\right)^{2}}{b Z \sqrt{1-\left(\frac{\lambda}{2 a}\right)^{2}}}[\mathrm{~dB} / \mathrm{m}],
$$

где $f$ - частота передаваемого сигнала, $\mathrm{Hz} ; \mu_{\alpha}-$ абсолютная магнитная проницаемость среды, $\mathrm{h} / \mathrm{m} ; \rho-$ удельное сопротивление материала скин-слоя, $\mathrm{Ohm} \cdot \mathrm{m}$; $a$ - ширина $(a>b)$ внутренней полости волновода, $\mathrm{m}$; $b$ - высота $(a>b)$ внутренней полости волновода, $\mathrm{m}$; $\lambda$ - длина волны передаваемого сигнала, $\mathrm{m} ; Z$ волновое сопротивление среды распространения сигнала, $\mathrm{Ohm}$.

Для удобства применения по полученным выражениям типа (2) построены универсальные диаграммы, определяющие значение коэффициента потерь при раз- 
личных сочетаниях исходных данных [5]. Однако, как указывают сами авторы $[5,6]$, действительные значения коэффициента потерь будут больше расчетных на 10-30\% вследствие неучтенных потерь на неплотности соединений, переходное сопротивление, лаковые покрытия и др.

\section{2. Моделирование нагрева волновода}

Процесс нагрева предлагается разбить на два этапа: разогрев тонкого скин-слоя при кратковременном сигнале и прогрев всей конструкции волновода при долговременной передаче сигналов СВЧ.

\section{1. Джоулево тепло}

Источником нагрева волновода является скин-слой (рис. 1) на его внутренних стенках, толщину $\delta$ которого можно определить по формуле [5]

$$
\delta=\frac{10^{3}}{2 \pi} \sqrt{\frac{10 \cdot \rho}{f \cdot \mu}}[\mathrm{m}],
$$

где $\rho$ - удельное сопротивление материала волновода, $\Omega \cdot \mathrm{m} ; f$ - частота передаваемого сигнала, $\mathrm{Hz}$; $\mu$ - относительная магнитная проницаемость материала скин-слоя:

$$
\mu=\frac{\mu_{a}}{\mu_{0}}
$$

где $\mu_{0}=4 \pi \cdot 10^{-7} \mathrm{H} / \mathrm{m}$ - магнитная проницаемость свободного пространства. $\mu_{a}-$ абсолютная магнитная проницаемость материала скин-слоя, $\mathrm{H} / \mathrm{m}$.

Для большинства применяемых для волноводов материалов (алюминий, медь, серебро и др.) толщины скинслоя весьма малы и имеют значения, которые несущественно изменяются во всем СВЧ диапазоне $3-30 \mathrm{GHz}$ (рис. 2). Поэтому в процессе передачи электромагнитного сигнала, в течение малого начального промежутка времени будет происходить интенсивный нагрев тонкого скин-слоя за счет протекания в нем наведенных токов.

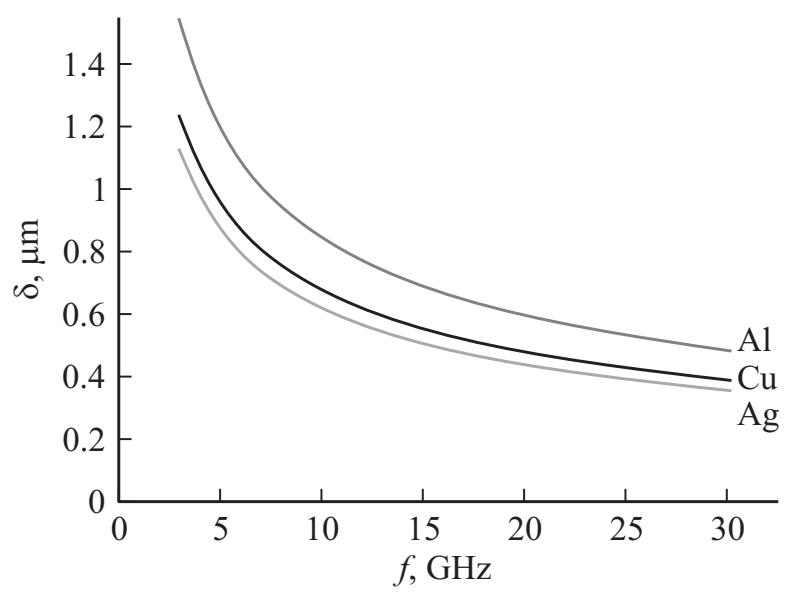

Рис. 2. Значение толщины скин-слоя в зависимости от материала для различных частот СВЧ сигнала.
Будем считать что потери мощности передаваемого по волноводу сигнала $\Delta P$ будут происходить только путем его преобразования в тепловую энергию. Данный процесс можно считать адиабатическим и его расчет можно проводить на основании закона Джоуля-Ленца [10], согласно которому

$$
I^{2} R d t=C M d T
$$

или

$$
\Delta P d t=C M d T .
$$

В итоге получим

$$
d T=\frac{\Delta P(t) d t}{C M}=\frac{\left(1-e^{-2 \alpha l}\right) P_{i n}(t) d t}{C M},
$$

где $T$ - температура нагрева, ${ }^{\circ} \mathrm{C} ; t-$ время нагрева, с; $C$ - удельная теплоемкость, $\mathrm{J} /\left(\mathrm{kg} \cdot{ }^{\circ} \mathrm{C}\right) ; M$ - масса тела, в котором выделяется мощность $P_{i n}, \mathrm{~kg}$.

Уравнение (6) получено для случая подводимой мощности $P_{i n}$, произвольно изменяющейся во времени $t$, что соответствует переменному электромагнитному сигналу. Если подводимую мощность усреднить и принять постоянной во времени (эффективная мощность $P_{\text {eff }}$ ), то выражение (6) примет вид

$$
\Delta T=\frac{\left(1-e^{-2 \alpha l}\right) P_{\mathrm{eff}} \Delta t}{C M}\left[{ }^{\circ} \mathrm{C}\right],
$$

где $\Delta T$ и $\Delta t-$ конечные изменения соответственно температуры и времени.

Полученные выражения (6) и (7) определяют линейную зависимость нагрева скин-слоя от времени и подводимой мощности.

\section{2. Уравнение теплопроводности}

По мере прогрева стенок волновода на его температурное поле начнет влиять теплообмен с окружающей средой, поэтому этот процесс уже нельзя считать адиабатическим. Разность температуры внутренних и наружных стенок приведет к возникновению удельного теплового потока через стенки волновода [10]

$$
\mathbf{q}=-\mathbf{n} \frac{d Q}{d t} \frac{1}{S}\left[\mathrm{~W} / \mathrm{m}^{2}\right]
$$

где $\mathbf{n}$ - единичный вектор нормали к поверхности $S$ в сторону увеличения температуры; $\frac{d Q}{d t}-$ количество тепла, проходящего в единицу времени, $\mathrm{W} ; S$ - площадь изотермической поверхности, $\mathrm{m}^{2}$.

Знак „минус“ означает, что вектор плотности теплового потока направлен в сторону уменьшения температуры и противоположно вектору нормали $\mathbf{n}$.

Плотность теплового потока q также можно определить, согласно основному закону теплопроводности Фурье, по формуле

$$
\mathbf{q}=-\mathbf{n} \lambda \frac{\partial T}{d n}
$$

где $\lambda$ - коэффициент теплопроводности, $\frac{\mathrm{W}}{\mathrm{m}^{\circ} \mathrm{C}} ; \frac{\partial T}{d n}-$ градиент температуры в направлении нормали $\mathbf{n}$. 


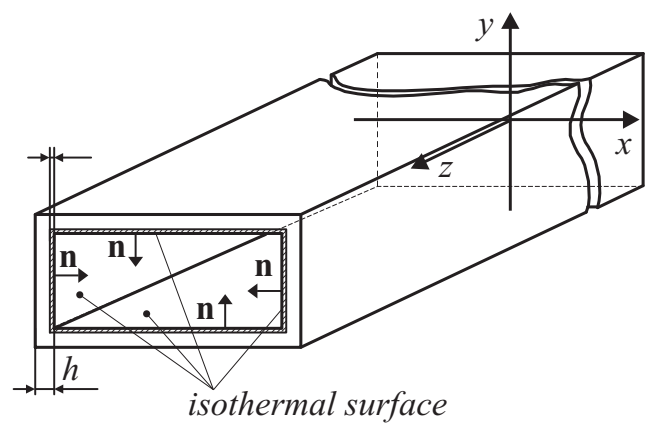

$a$

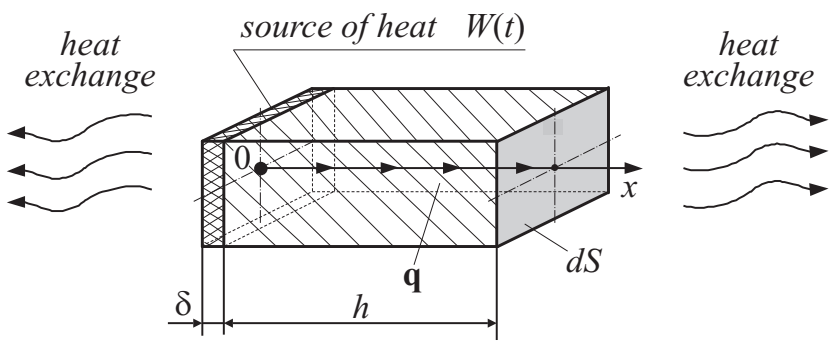

Pис. 3. Расчетная схема нагрева: $a-$ волновод, $b-$ элемент $d S$ стенки волновода (одномерная модель).

Примем еще одно допущение: при передаче электромагнитного сигнала по волноводу происходит равномерный разогрев всех поверхностей его внутренних стенок, которые в результате будут представлять собой изотермическую поверхность $S$ (рис. 3,a), значение которой можно определить как

$$
S=2 l(a+b)\left[\mathrm{m}^{2}\right] .
$$

Направление нормали $\mathbf{n}$ к изотермической поверхности будет перпендикулярно к каждой из стенок волновода. Следовательно, распространение тепла в волноводе при передаче электромагнитного сигнала будет происходить только в одном направлении - по нормали $\mathbf{n}$ в сторону наружных стенок (рис. 3,a). Краевыми эффектами в углах соединения пластин будем пренебрегать.

Принятый нами подход позволяет принять для расчета температурного поля волновода одномерную модель распространения тепла в виде эквивалентного стержня (рис. $3, b$ ), имеющего поперечное сечение площадью $S(10)$.

Выделение тепла $\Delta Q$ в скин-слое при прохождении по нему электромагнитного сигнала можно смоделировать источником тепла интенсивностью $W(t)$.

Тогда для моделирования нагрева волновода будет справедливо одномерное параболическое дифференциальное уравнение теплопроводности с источником тепла в виде [10]

$$
\frac{\partial T(x, t)}{\partial t}=a_{2} \frac{\partial^{2} T(x, t)}{\partial x^{2}}+\frac{W(t)}{c_{1} \gamma_{1}}
$$

где $a_{2}-$ коэффициент температуропроводности материала стенок волновода, который определяется по формуле

$$
a_{2}=\frac{\lambda_{2}}{c_{2} \gamma_{2}}\left[\frac{\mathrm{m}^{2}}{\mathrm{c}}\right]
$$

в которой $\lambda_{2}$ - коэффициент теплопроводности материала стенок, $\frac{\mathrm{W}}{\mathrm{m} \cdot{ }^{\circ} \mathrm{C}} ; c_{2}-$ удельная теплоемкость материала стенок, $\frac{\mathrm{J}}{\mathrm{kg} \cdot{ }^{\circ} \mathrm{C}} ; \gamma_{2}-$ удельная плотность материала стенок, $\frac{\mathrm{kg}}{\mathrm{m}^{3}} \cdot c_{1}-$ удельная теплоемкость материала скин-слоя, $\frac{\mathrm{J}}{\mathrm{kg} \cdot{ }^{\circ} \mathrm{C}} ; \gamma_{1}-$ удельная плотность материала скин-слоя, $\frac{\mathrm{kg}}{\mathrm{m}^{3}} ; W(t)-$ интенсивность источника тепла в скин-слое, $\left[\frac{\mathrm{W}}{\mathrm{m}^{3}}\right]$ :

$$
W(t)=\frac{\Delta P(t)}{V}=\frac{\Delta P(t)}{S \cdot \delta}=\frac{\left(1-e^{-2 \alpha l}\right) P_{i n}(t)}{S \cdot \delta} .
$$

Для получения однозначного решения дифференциального уравнения (11) необходимо задать краевые условия, которые включают начальное и граничные условия на наружной и внутренней стенках волновода.

\section{3. Краевые условия}

В качестве начального условия можно принять однородное температурное поле, которое для одномерной модели запишется в виде равенства температуры во всех точках волновода некоторой заданной величине в начальный момент времени $t=0$ :

$$
T(x, t=0)=T_{0}=\text { const. }
$$

Граничные условия задаются на границах рассматриваемой области, ими в рассматриваемом случае будут являться внутренняя и наружная поверхности стенки волновода (рис. 3), на которых будет происходить конвективный теплообмен с окружающей средой, имеющей температуру $T_{\text {surr }}$ (граничные условия 3-го рода).

Для внутренней поверхности стенки $(x=0)$ условие теплообмена будет иметь вид

$$
\lambda_{1} \frac{\partial T_{1}(x=0, t)}{\partial x}+\alpha_{1}\left[T_{1}(x=0, t)-T_{\text {surr } 1}\right]=0,
$$

где $T_{l}(x=0, t)$ - температура на поверхности внутренней стенки волновода; $\alpha_{l}-$ коэффициент теплообмена поверхности внутренней стенки со средой внутри волновода, $\frac{\mathrm{W}}{\mathrm{m}^{2} \cdot{ }^{\circ} \mathrm{C}} ; T_{\text {surr1 }}-$ температура среды внутри волновода, ${ }^{\circ} \mathrm{C}$.

Второе граничное условие определяет условие теплообмена наружных стенок волновода $(x=h)$ с окружающей средой и имеет вид

$$
\lambda_{2} \frac{\partial T_{2}(x=h, t)}{\partial x}+\alpha_{2}\left[T_{2}(x=h, t)-T_{\text {surr }}\right]=0,
$$

где $T(x=h, t)$ - температура на внешней поверхности стенок волновода; $\alpha_{2}-$ коэффициент теплообмена внешних поверхностей стенок с окружающей средой 
снаружи волновода, $\frac{\mathrm{W}}{\mathrm{m}^{2} \cdot{ }^{\circ} \mathrm{C}} ; T_{\text {surr2 }}-$ температура окружающей среды снаружи волновода, ${ }^{\circ} \mathrm{C}$.

Граничными условиями (13), (14) в качестве основного способа теплообмена с окружающей средой принимается конвекция, что соответствует работе волновода в среде газа (воздуха), т. е. на Земле.

Условия эксплуатации волноводов в составе космического аппарата связи, имеющего открытую платформу, на заданной орбите предполагают, что они будут функционировать в среде вакуума, в котором конвекция практически отсутствует, а преобладающим способом переноса и рассеивания тепловой энергии конструкции волноводов будет являться лучистый теплообмен.

В этом случае вместо условия конвекции (14) на внешних поверхностях стенок волновода необходимо задать условие лучистого теплообмена, которое согласно закону Стефана-Больцмана имеет вид заданного теплового потока

$$
\lambda_{2} \frac{\partial T_{2}(x=h, t)}{\partial x}-\sigma_{2}\left[T_{\text {surr } 2}^{4}-T_{2}^{4}(x=h, t)\right]=0,
$$

где $\sigma_{2}-$ приведенный коэффициент лучеиспускания для наружных стенок волновода, $\frac{\mathrm{W}}{\mathrm{m}^{2} \cdot{ }^{\circ} \mathrm{C}}$.

Анализ распространения тепла в волноводе показывает, что его внутренние стенки представляют собой замкнутую поверхность из ортогонально расположенных пластин, между которыми за счет лучистого теплообмена будет происходить взаимный разогрев, который будет носить сложный нелинейный характер. Поэтому с достаточной для практических расчетов точностью для внутренних стенок волновода можно принять условие отсутствия теплообмена с окружающей средой, которое запишется в виде

$$
\frac{\partial T_{1}(x=0, t)}{\partial x}=0 .
$$

Особенностью эксплуатации открытых конструкций волноводов будет являться зависимость их теплового состояния от их расположения относительно Солнца: в тени волноводы будут излучать тепло в открытое космическое пространство, имеющее меньшую температуру $\left(T_{\text {surr2 }}<T_{2}(x=h, t)\right)$, и будет справедливо граничное условие (15). В случае же расположения конструкции волновода под прямыми солнечными лучами будет происходить его нагрев, поскольку в этом случае температура окружающей среде может быть больше температуры стенок $\left(T_{\text {surr } 2}>T_{2}(x=h, t)\right)$.

Полученное дифференциальное уравнение (11) с краевыми условиями (12)-(15) позволяет определять температурные поля прямолинейного участка волновода для любых встречающихся на практике его размеров, материала и характеристик СВЧ сигнала, для которых известен коэффициент потерь $\alpha$.

Способы решения дифференциального уравнения (11) с заданными начальными (12) и граничными условиями (13)-(15) к настоящему времени хорошо изучены [11] и его можно решить как аналитическими методами (метод разделения переменных, метод источников и др.), так и численными (метод конечных разностей, метод конечных элементов и др.).

Тепловой расчет волноводов с криволинейной продольной осью затруднен прежде всего сложностью выбора значений коэффициентов потерь $\alpha$, поскольку для этих случаев его значения в известной литературе [5-9] не приводятся и в данной работе не рассматриваются. Однако в последнее время появляются работы, в которых численными методами выполняются исследования распространения электромагнитных полей и в криволинейных волноводах [12].
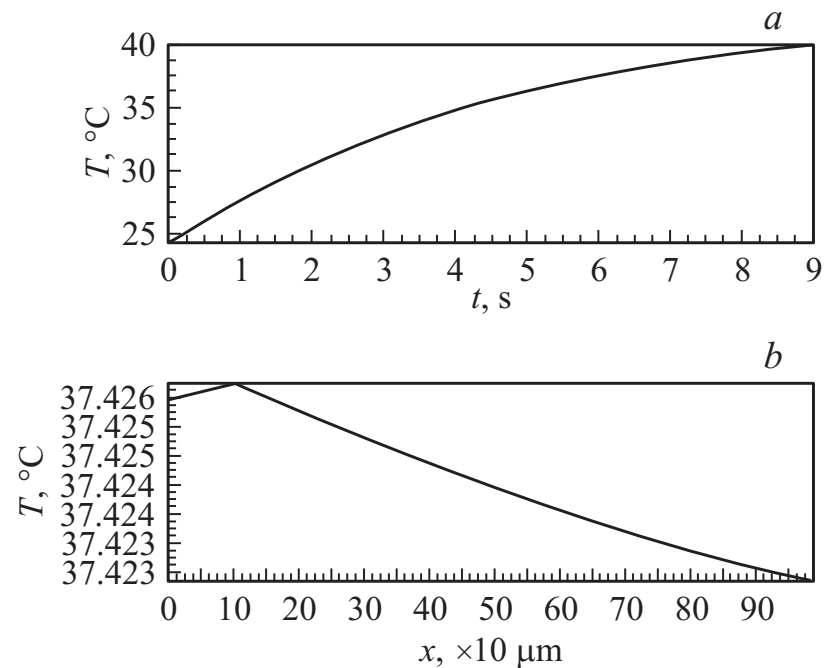

Рис. 4. Результаты расчета температурных полей при передаче СВЧ сигнала по волноводу при наличии конвекции: $a-$ зависимость температуры скин-слоя от времени, $b-$ распределение температуры по толщине стенки.
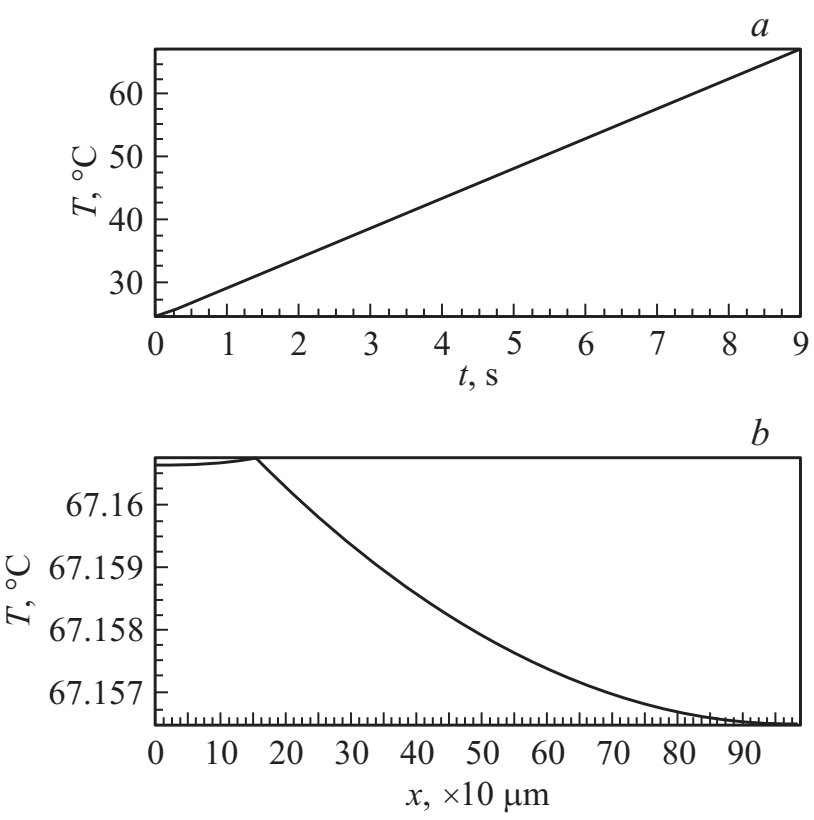

Рис. 5. То же, что на рис. 4, при отсутствии конвекции. 


\section{3. Результаты расчета}

Согласно разработанной модели и полученным уравнениям (11)-(15), проведены расчеты температурного поля для прямых участков волноводов с различным типоразмером поперечного сечения и толщиной стенки. Решение выполнялось аналитическим методом разделения переменных и численным методом конечных разностей по явной схеме в разработанной для этого программах $[13,14]$.

Изучалось время нагрева скин-слоя и установления стационарного температурного поля стенок волновода в двух вариантах: при наличии конвекции (работа волновода в атмосфере Земли) и без нее (работа волновода в вакууме).

Результаты расчетов показывают, что из-за малой толщины скин-слоя время его нагрева очень мало (< $1 \mathrm{~s})$, что обосновывает важность учета влияния даже кратковременной передачи СВЧ сигналов высокой мощности на условия термоусталостной прочности материала скин-слоя.

Время установления второго этапа нагрева, при котором образуется стационарное температурное поле для всей конструкции волновода, определяемое уравнением (11) с краевыми условиями (12)-(14), вследствие высокой теплопроводности и низкой удельной теплоемкости материала волновода (алюминий, медь и др.) также весьма мало, и продолжительная (> $1 \mathrm{~s}$ ) передача СВЧ сигналов высокой мощности будет оказывать влияние на его общее температурное состояние.

Например, на рис. 4 и 5 представлены результаты расчета температурных полей при передачи СВЧ сигнала частотой $10 \mathrm{GHz}$ и мощностью $10 \mathrm{~kW}$ по волноводу из алюминиевого сплава АД31 сечением $15 \times 35 \mathrm{~mm}$.

Установлено, что на интенсивность нагрева волновода существенное влияние оказывают граничные условия. Так, учет даже незначительной конвекции (рис. 4) вследствие большой площади наружной поверхности волновода существенно понижает величину его установившейся температуры. При моделировании условий эксплуатации космического аппарата на орбите (15), (16) в условиях отсутствия конвекции нагрев волноводов происходит значительно быстрее и на большие значения температур (рис. 5).

Таким образом, полученные результаты показывают, что для оценки статического и динамического напряженно-деформированного состояний протяженных пространственно-разветвленных тонкостенных конструкций волноводно-распределительных систем космических аппаратов связи на орбите требуется учет совместного действия всех вышеуказанных причин нагрева.

\section{4. Заключение}

Разработаны математические модели процесса нагрева волновода и получено разрешающее дифференциальное уравнение задачи с начальными и граничными условиями, соответствующие условиям работы волноводов на Земле и в открытом космосе.
По разработанным методам и полученным зависимостям разработана программа расчета на ЭВМ тепловых полей и учета их влияния на напряженно-деформированное состояние волноводов антенно-фидерных систем космических аппаратов связи.

Выполненные расчеты показали, что существующие методы расчета статической и динамической прочности волноводов требуют уточнения на предмет учета влияния их нагрева от передачи СВЧ сигналов высокой мощности и солнечного излучения.

На основе полученных результатов рассматривается возможность разработки методов для расчета усталостной прочности материала скин-слоя волноводов.

Работа выполнена при поддержке гранта президента РФ № МК-2875.2015.8 и гранта РФФИ 16-48-242110 р_офи_м.

\section{Список литературы}

[1] Silchenko P.N., Mikhnev M.M., Ankudinov A.V., Kudryavtsev I.V. // J. Machinery Manufacture and Reliability. 2012. Vol. 41. № 1. P. 91-95.

[2] Сильченко П.Н., Кудрявцев И.В., Михнёв М.М., Наговиuин В.Н. // Журн. СФУ. Сер.: Техника и технологии. 2012. № 2. C. $150-161$.

[3] Сильченко П.Н., Кудрявцев И.В., Михнёв М.М., Халиманович В.И., Наговицин В.Н. // Журн. СФУ. Сер.: Техника и технологии. 2012. № 2. С. 205-219.

[4] Трощенко В.T. Сопротивление усталости металлов и сплавов. 1987. 1238 с.

[5] ЕФимов И.Е. Волновые линии передачи. 1979. 232 с.

[6] Кинг Р. Передающие линии, антенны, волноводы. 2012. $360 \mathrm{c}$.

[7] Воскресенский Д.И. и др. Устройства СВЧ и антенны. 2006. $378 \mathrm{c}$.

[8] Пименов Ю.В. и др. Техническая электродинамика. 2000. $536 \mathrm{c}$.

[9] Котельников И.А. О затухании в волноводе // ЖТФ. 2004. т. 74. Вып. 9. С. 91-96.

[10] Карташов Э.М. Аналитическая теория теплопроводности и прикладной термоупругости. 2012. 656 с.

[11] Голоскоков Д.П. Уравнения математической физики. Решения задач в системе Maple. 2004. 539 с.

[12] Finnveden $S$. Waveguide finite elements for curved structures / J. Sound and Vibration. 2008. № 312. P. 644-671.

[13] Кудрявцев И.В., Горелюк О.Б., Новиков Е.С., Кудрявцева Ю.М. Программа для расчета теплового состояния волноводов при передаче СВЧ-сигнлов высокой мощности ThermoLoss. Свидетельство о государственной регистрации программы для ЭВМ № 201660369.2016 // Официальный бюллетень Реестра программ для ЭВМ. M., 14.09.2016 г.

[14] Кудрявщев И.В., Горелюк О.Б., Кудрявщева Ю. Программа для оценки потерь мощности СВЧ-сигналов при передаче по прямым участкам волноводов „PowerLoss“. Свидетельство о государственной регистрации программы для ЭВМ № 2016660485. 2016 // Официальный бюллетень Реестра программ для ЭВМ. М., 15.09.2016 г. 